\title{
The effect of simultaneous and sequential presentation of stimulus dimensions on absolute judgment accuracy*
}

\author{
JOHN H. FLOWERS \\ University of Nebraska, Lincoln, Nebraska 68508
}

\begin{abstract}
The effects of spatial stimulus repetition, sequential stimulus repetition, spatially separated dimensional redundancy, and sequentially presented dimensional redundancy on absolute judgment accuracy of hue and brightness were compared. Two exposure durations, 0.1 and $2.0 \mathrm{sec}$, were used. While spatial repetition did not improve accuracy for either dimension, the sequential repetition of brightness produced a small increase in accuracy. The spatial presentation of correlated values of both dimensions increased accuracy only at the $2.0-\sec$ duration. The sequential presentation of both dimensions increased accuracy, but only at the 2.0 -sec duration was this gain substantial and greater than that provided by the sequential repetition of brightness alone.
\end{abstract}

Stimulus redundancy exists when more stimulus information is provided than is necessary for the number of discriminations required in a psychological task. During the past 25 years, psychologists have examined the effects of various forms of stimulus redundancy upon the ability of both animals and humans to discriminate among stimuli. Unfortunately, as Garner (1972) points out, much of this research has led to conflicting conclusions. The present study is directed at answering several questions raised in previous research concerning the ability of human observers to combine perceptual information from separately presented stimulus dimensions.

\section{Separable Dimensions and Perceptual Performance}

Dimensional redundancy occurs when different stimulus dimensions, such as size, brightness, or hue, are correlated within a set of stimuli. These redundant dimensions may be compounded into the same stimulus element or they may be presented in sequentially or spatially discrete elements. Most studies have used the compounded variety (e.g., Munsell color patches varying in correlated combinations of size, hue, brightness, or saturation) and have, in general, shown that compounded dimensional redundancy is effective in increasing accuracy of stimulus identification (Eriksen \& Hake, 1955; Lockhead, 1966a) and speed of discrimination (e.g., Garner \& Felfoldy, 1970).

While Garner and Felfoldy (1970) demonstrated that the compounding of correlated values of brightness and saturation of Munsell colors in single stimulus patches increased speed of card sorting, these authors also showed that the correlation of these dimensions in

*This research was supported by Grant MH 14229 from the National Institute of Mental Health to Yale University, and this paper is based upon a doctoral dissertation presented to the faculty of Yale University. The author wishes to thank W. R. Garner for his guidance throughout this research, and J. C. Stevens, W. Kessen, A. J. Wearing, and T. L. Powley for their thoughtful criticism. spatially separated patches produced no increase in discrimination speed. Garner and Felfoldy argued that brightness and saturation, when compounded, were integral dimensions which were not separately encoded, but were combined to form a more effective discrimination continuum. Garner and Felfoldy maintained that these same dimensions, when presented in separate stimulus elements, were perceptually separable dimensions which would have to be processed individually. Separable dimensions should not, therefore, increase speed of discrimination when combined in a correlated manner.

Lockhead (1966b) demonstrated that the presentation of redundant, but obviously separable, stimulus dimensions might increase accuracy in an absolute judgment task. Lockhead found that the sequential presentation of Munsell hues and grays, having correlated values of hue and brightness, produced greater absolute judgment accuracy than that obtained when only a single dimension was relevant. Lockhead noted, however, that the magnitude of this gain in accuracy was considerably less than that obtained in tasks in which hue and brightness were compounded into the same color patch (Eriksen \& Hake, 1955). Lockhead therefore suggested that the increase in accuracy which resulted from the sequential presentation of hue and brightness might have resulted from multiple opportunities over time to perceive the same perceptual information, rather than the integration of perceptual information from the two dimensions. Lockhead thus hypothesized that temporal repetition of identical stimuli might produce an equal gain in accuracy. A comparison of the effects of sequential dimensional redundancy with the effects of simple temporal stimulus repetition was one goal of the present study.

Lockhead (1966b) also presented the correlated dimensions of hue and brightness in spatially separated patches which were simultaneously exposed in a single field. This form of spatially separated dimensional redundancy produced no greater accuracy than the 
better single dimension for each $\mathrm{S}$. Similar results have been obtained for the simultaneous presentation of brightness and loudness (e.g., Lockhead, 1970). Thus, one might conclude that separable redundant stimulus dimensions must be sequentially presented in order to increase accuracy of identification. In Lockhead's (1966b) study, in which the spatially separated presentation of hue and brightness failed to increase information transmission, the stimulus field was exposed for only $0.1 \mathrm{sec}$. This duration was probably much too brief to permit eye fixation on both stimulus patches. Furthermore, any integration strategy involving the "averaging" of separately encoded responses (see Garner \& Morton, 1969) would probably be precluded by the brief exposure duration, particularly if such a strategy required the serial processing of each stimulus dimension. In the condition involving sequential presentation of hue and brightness, each dimension was presented for $0.1 \mathrm{sec}$ with a 0.5 -sec delay between fields. While this condition may have permitted foveal fixation on each dimension, it is still possible that the brief durations may have placed time limitations on potentially useful integration strategies. The effects of exposure duration on the efficiency with which Ss can integrate information from separately presented correlated stimuli are therefore investigated in the present study.

\section{Purpose}

The present study is a comparison of the effects of spatial stimulus repetition, sequential stimulus repetition, spatially separated dimensional redundancy, and sequentially presented dimensional redundancy on absolute judgment accuracy. To determine if processing time limits the efficiency with which these forms of redundancy might increase discrimination accuracy, two exposure durations are used.

\section{METHOD}

\section{Subjects}

Four male graduate students in the Yale psy chology department were paid $\$ 80$ for participation in 12 experimental sessions, lasting between 2.5 and $3.0 \mathrm{~h}$ each, plus a 1-h introductory session (given to familiarize $S$ s with the absolute judgment task and to determine if any $S$ had a visual defect which would make him unable to participate). Each $S$ was run three times per week; the time span of the experiment was 4 weeks for each $S$.

\section{Stimulus Materials}

Stimulus materials consisted of squares of Munsell paper, $2.54 \mathrm{~cm}$ on a side. These squares were mounted on rectangular white cards, which were approximately $12.7 \times 17.8 \mathrm{~cm}$. Viewing distance was approximately $1.09 \mathrm{~m}$. Two sets of Munsell paper were used, corresponding to the dimensions of brightness and hue. The brightness series consisted of 15 Munsell grays, N2 to N9 in Munsell value notation, in steps of .5. These 15 different brightness levels, which, in order of increasing brightness, correspond to the stimulus categories " 1 " through "15," respectively. The hue series consisted of 15 squares of Munsell paper, each of Munsell Value 5 and Chroma 6, ranging in Munsell hue notation from $2.5 \mathrm{GY}$ to $7.5 \mathrm{~B}$ in steps of 2.5 . These hue levels corresponded to the stimulus categories "1" to "15," respectively.

\section{Task}

The task was one of absolute judgment, using 15 response categories labeled 1-15. The Ss were run individually and made judgments of eight sets of stimuli during an experimental session. Each of these eight sets of stimuli required 60 responses, and corresponded to one of the eight stimulus conditions described below. Within each set of 60 stimuli, each of the 15 stimulus categories was presented four times, in a random order, with the restriction that no stimulus category could be repeated on successive trials. The Ss were told of this restriction. Each $S$ therefore contributed 480 recorded observations per experimental session.

The experiment was conducted in a dimly lit room. The Ss were given about $4 \mathrm{~min}$ to dark-adapt before beginning the experiment.

Before beginning a set of 60 trials, Ss were given 10 to 20 warm-up trials, for which responses were not recorded. Following each set of 60 trials, Ss were permitted a short break.

Stimuli were displayed in a Scientific Prototype Model GB tachistoscope. Each trial was initiated by $S$, by pressing a telegraph key. Prior to the depression of the key, a fixation field containing a small cross in the center was present. When the key was depressed, a blank field appeared for $0.5 \mathrm{sec}$, followed by the stimulus field. The fixation, blank, and stimulus fields were of equal brightness (about $29 \mathrm{fL}$ ). Following each stimulus presentation, $S$ gave an oral response, which was recorded by $E$. The $E$ then orally informed $S$ of the correct response, and placed the next stimulus in the tachistoscope. Ss were self-paced and quickly developed a pattern of allowing about $1 \mathrm{sec}$ between the placement of the next stimulus and the next keypress.

\section{Stimulus Conditions}

There were eight stimulus conditions.

\section{Hue Alone (H) and Brightness Alone (B)}

In these two conditions, Ss were exposed, on each trial, to a single Munsell patch. In Condition $\mathrm{H}$, each patch was from the hue series, while in Condition $B$, each patch was from the brightness series. The center of each patch was always located $2.54 \mathrm{~cm}$ to the left of the geometric center of the stimulus field, or about $1.5 \mathrm{deg}$ of visual angle from the center of the fixation field. $\mathbf{H}$ and $B$ were nonredundant control conditions.

\section{Hue Repeated Simultaneously (HH sim) and Brightness Repeated Simultaneously (BB sim)}

In these two conditions, Ss viewed, on each trial, two identical patches centered $2.54 \mathrm{~cm}$ to the right and left, respectively, of the center of a stimulus field. Both patches were exposed simultaneously. In Condition HH sim, the patches were from the hue series, while in Condition BB sim, the patches were from the brightness series.

\section{Hue Repeated Sequentially (HH seq) and Brightness Repeated Sequentially (BB seq)}

In these two conditions, Ss viewed, on each trial, two stimulus fields, separated by an 0.5 -sec blank field between the offset of the first field and the onset of the second. The first field contained a single Munsell patch, which was located $2.54 \mathrm{~cm}$ to the left of the center of the field. The second field contained an 
Table 1

Accuracy of Absolute Judgment

\begin{tabular}{|c|c|c|c|c|c|c|c|c|}
\hline \multirow[b]{2}{*}{ Ss } & \multicolumn{8}{|c|}{ Conditions } \\
\hline & $\mathbf{H}$ & $\mathrm{HH}$ sim & HH seq & B & BB sim & BB seq & HB sim & HB seq \\
\hline \multicolumn{9}{|c|}{ 0.1-Sec Duration } \\
\hline J.P. & $\begin{array}{r}47.7^{*} \\
(2.22)\end{array}$ & $\begin{array}{c}50.0 \\
(2.29)\end{array}$ & $\begin{array}{c}53.7 \\
(2.29)\end{array}$ & $\begin{array}{c}50.3 \\
(2.15)\end{array}$ & $\begin{array}{c}48.3 \\
(2.27)\end{array}$ & $\begin{array}{c}55.0 \\
(2.34)\end{array}$ & $\begin{array}{c}52.3 \\
(2.29)\end{array}$ & $\begin{array}{c}53.0 \\
(2.40)\end{array}$ \\
\hline D.H. & $\begin{array}{c}39.3 \\
(1.94)\end{array}$ & $\begin{array}{c}39.7 \\
(2.06)\end{array}$ & $\begin{array}{c}37.7 \\
(2.09)\end{array}$ & $\begin{array}{c}47.0 \\
(2.17)\end{array}$ & $\begin{array}{c}46.3 \\
(2.13)\end{array}$ & $\begin{array}{c}54.0 \\
(2.33)\end{array}$ & $\begin{array}{c}43.3 \\
(2.12)\end{array}$ & $\begin{array}{c}49.7 \\
(2.28)\end{array}$ \\
\hline G.K. & $\begin{array}{c}58.3 \\
(2.49)\end{array}$ & $\begin{array}{c}58.3 \\
(2.49)\end{array}$ & $\begin{array}{c}65.3 \\
(2.66)\end{array}$ & $\begin{array}{c}61.7 \\
(2.57)\end{array}$ & $\begin{array}{c}58.3 \\
(2.53)\end{array}$ & $\begin{array}{c}69.7 \\
(2.75)\end{array}$ & $\begin{array}{c}66.7 \\
(2.65)\end{array}$ & $\begin{array}{c}67.0 \\
(2.70)\end{array}$ \\
\hline J.A. & $\begin{array}{c}42.3 \\
(2.13)\end{array}$ & $\begin{array}{c}48.0 \\
(2.13)\end{array}$ & $\begin{array}{c}50.0 \\
(2.26)\end{array}$ & $\begin{array}{c}52.3 \\
(2.34)\end{array}$ & $\begin{array}{c}50.7 \\
(2.31)\end{array}$ & $\begin{array}{c}56.3 \\
(2.47)\end{array}$ & $\begin{array}{c}56.7 \\
(2.44)\end{array}$ & $\begin{array}{c}59.3 \\
(2.47)\end{array}$ \\
\hline Mean & $\begin{array}{c}46.9 \\
(2.19)\end{array}$ & $\begin{array}{c}49.0 \\
(2.24)\end{array}$ & $\begin{array}{c}51.7 \\
(2.33)\end{array}$ & $\begin{array}{c}52.8 \\
(2.31)\end{array}$ & $\begin{array}{c}50.9 \\
(2.31)\end{array}$ & $\begin{array}{c}58.8 \\
(2.48)\end{array}$ & $\begin{array}{c}54.7 \\
(2.37)\end{array}$ & $\begin{array}{c}57.3 \\
(2.46)\end{array}$ \\
\hline \multicolumn{9}{|c|}{ 2.0-Sec Duration } \\
\hline J.P. & $\begin{array}{c}58.7 \\
(2.46)\end{array}$ & $\begin{array}{c}63.0 \\
(2.52)\end{array}$ & $\begin{array}{c}61.7 \\
(2.45)\end{array}$ & $\begin{array}{c}61.0 \\
(2.54)\end{array}$ & $\begin{array}{c}64.3 \\
(2.63)\end{array}$ & $\begin{array}{c}62.0 \\
(2.55)\end{array}$ & $\begin{array}{c}70.3 \\
(2.95)\end{array}$ & $\begin{array}{c}72.7 \\
(2.99)\end{array}$ \\
\hline D.H. & $\begin{array}{c}55.7 \\
(2.50)\end{array}$ & $\begin{array}{l}60.7 \\
(2.49)\end{array}$ & $\begin{array}{c}59.3 \\
(2.48)\end{array}$ & $\begin{array}{c}62.3 \\
(2.56)\end{array}$ & $\begin{array}{c}61.0 \\
(2.58)\end{array}$ & $\begin{array}{c}65.3 \\
(2.61)\end{array}$ & $\begin{array}{c}66.3 \\
(2.70)\end{array}$ & $\begin{array}{c}69.7 \\
(2.87)\end{array}$ \\
\hline G.K. & $\begin{array}{c}63.7 \\
(2.66)\end{array}$ & $\begin{array}{c}66.0 \\
(2.71)\end{array}$ & $\begin{array}{c}60.7 \\
(2.58)\end{array}$ & $\begin{array}{c}58.7 \\
(2.51)\end{array}$ & $\begin{array}{l}61.3 \\
(2.60)\end{array}$ & $\begin{array}{c}69.3 \\
(2.75)\end{array}$ & $\begin{array}{c}67.0 \\
(2.76)\end{array}$ & $\begin{array}{c}71.0 \\
(2.87)\end{array}$ \\
\hline J.A. & $\begin{array}{c}49.7 \\
(2.35)\end{array}$ & $\begin{array}{c}53.7 \\
(2.34)\end{array}$ & $\begin{array}{c}53.3 \\
(2.33)\end{array}$ & $\begin{array}{c}51.7 \\
(2.30)\end{array}$ & $\begin{array}{c}53.3 \\
(2.28)\end{array}$ & $\begin{array}{c}61.0 \\
(2.54)\end{array}$ & $\begin{array}{c}53.0 \\
(2.34)\end{array}$ & $\begin{array}{c}63.3 \\
(2.72)\end{array}$ \\
\hline Mean & $\begin{array}{c}56.9 \\
(2.49)\end{array}$ & $\begin{array}{c}60.8 \\
(2.52)\end{array}$ & $\begin{array}{c}58.8 \\
(2.46)\end{array}$ & $\begin{array}{c}58.4 \\
(2.48)\end{array}$ & $\begin{array}{l}60.0 \\
(2.52)\end{array}$ & $\begin{array}{c}64.4 \\
(2.63)\end{array}$ & $\begin{array}{c}64.2 \\
(2.69)\end{array}$ & $\begin{array}{c}69.2 \\
(2.86)\end{array}$ \\
\hline
\end{tabular}

*Main cell entries are hit rate in percent correct; parenthesized entries are information transmission values in bits.

identical patch located $2.54 \mathrm{~cm}$ to the right of the center of the field. In Condition $\mathrm{HH}$ seq, the patches were from the hue series, while in Condition BB seq, the patches were from the brightness series.

\section{Hue and Brightness Presented Simultaneously (HB sim)}

In this condition, Ss viewed a single stimulus field on each trial. For half the $S s$, the field contained a brightness patch located $2.54 \mathrm{~cm}$ to the left of the center of the field and the corresponding hue patch located $2.54 \mathrm{~cm}$ to the right of the center of the field. For the remaining Ss, these positions were reversed.

\section{Hue and Brightness Presented Sequentially (HB seq)}

In this condition, two stimulus fields were presented on each trial. For half the Ss, the first field contained a hue patch located $2.54 \mathrm{~cm}$ to the left of the center of the field, while the second field contained the corresponding brightness patch $2.54 \mathrm{~cm}$ to the right of the center of the field. For the remaining Ss, the brightness patch appeared first, to the left of center, followed by the hue patch on the right. The second field was separated from the first by an 0.5 -sec blank field.

\section{Exposure Duration}

Each of the eight stimulus conditions described above, was presented at two exposure durations in order to provide two levels of processing time. These durations were 0.1 and $2.0 \mathrm{sec}$. In the conditions in which two fields were presented on each trial (HH seq, BB seq, HB seq), each of these two fields was presented for either 0.1 or $2.0 \mathrm{sec}$.

\section{Instructions to Subjects}

The Ss were not given suggestions about specific judgment strategies, but were told to use whatever strategy they wished in any of the conditions, as long as they felt that the strategy chosen provided the greatest accuracy possible. The Ss were told that the purpose of the fixation cross was only to establish depth of focus, and that fixation upon the center of each patch (which was always located either to the right or left of the center of the field) was permissible. The Ss were also told to proceed at their own pace, and that there was no obligation to respond quickly following the stimulus presentation.

\section{Order of Presentation of Conditions}

Two of the Ss received each of the eight stimulus conditions with the $0.1-\sec$ exposure duration in each of six experimental sessions, followed by six sessions in which the 2.0 -sec duration was used. For the remaining Ss, the $2.0-\mathrm{sec}$ duration was given in the first six sessions, followed by six sessions in which the 0.1 -sec duration was used. Eight different orders of presenting stimulus conditions (determined by an 8 by 8 Latin square) within each session were used. In each of these orders, each of the eight stimuli occurred once. The Ss were assigned to a different order in each session, to minimize confounding of fatigue or practice effects with experimental conditions.

The first and seventh experimental sessions (i.e., the first sessions in which a particular exposure duration was used) were considered practice sessions and were omitted from analysis. Thus, each $\mathrm{S}$ contributed 300 observations per stimulus condition for each of the two exposure durations.

\section{RESULTS}

Two measures of discrimination accuracy were calculated, hit rate (HR) and information transmission (IT). ${ }^{1}$ Mean HR and IT values for each $S$ are presented in Table 1. Analysis of variance of HR showed an increase in accuracy over sessions, $F(4,12)=15.6$, $p<.001$, but the Sessions by Conditions interaction was 
not significant $(F<1)$. Analysis of variance of both HR and IT indicated a strong effect of stimulus conditions, $F(7,21)=16.2, p<.001$ for $\mathrm{HR}$, and $F(7,21)=18.98$, $\mathrm{p}<.001$ for IT. 2 Mean HR and IT values for each $\mathrm{S}$ are presented in Table 1.

\section{Spatial Stimulus Repetition}

The effect of spatial stimulus repetition can be evaluated by comparing $\mathrm{HH}$ sim with $\mathrm{H}$ and $\mathrm{BB}$ sim with B. Neither comparison indicates any important improvement in accuracy with spatial repetition. While $\mathrm{HR}$ for $\mathrm{HH}$ sim is actually slightly greater than for $\mathrm{H}$ for $3 / 4$ Ss at the 0.1-sec duration and $4 / 4$ Ss at the 2.0-sec duration, accuracy as measured by IT was greater for only $2 / 4$ Ss at each duration. Similarly, there was very little difference in accuracy between B and BB sim as measured by either HR or IT.

\section{Sequential Stimulus Repetition}

The effects of sequential stimulus repetition can be evaluated by comparing $\mathrm{BB}$ seq with $\mathrm{H}$ and $\mathrm{BB}$ seq with B. At both the 0.1- and the 2.0-sec durations, BB seq produced greater accuracy than B for all four Ss as measured either by HR or $1 \mathrm{IT}^{3}$ At the $0.1-\mathrm{sec}$ duration, HR for BB seq was greater than for B in 16/20 sessions (5/5 sessions for D.H. and J.P. and $3 / 5$ sessions for the other two Ss). At the 2.0-sec duration, HR for BB seq was greater than for $B$ in $16 / 20$ sessions $(5 / 5$ sessions for J.A., $4 / 5$ sessions for G.K. and D.H., and $3 / 5$ sessions for J.P.). While the mean amount of improvement in IT (.19 and .15 bits for the 0.1 - and $2.0-\mathrm{sec}$ durations, respectively) is small in comparison to those obtained in previous experiments (e.g., Eriksen \& Hake, 1955) in which correlated stimulus dimensions were compounded into the same element, it is very close to the gain accuracy reported by Lockhead (1966b) for the sequential presentation of separate hue and brightness patches.

On the other hand, the data do not indicate any consistent improvement in accuracy with the sequential repetition of hue. While the mean gain in accuracy of $4.9 \%$ in HR and .14 bits in IT might suggest a small amount of facilitation, it should be noted that HR for $\mathrm{HH}$ seq was greater than for $\mathrm{H}$ for only $3 / 4 \mathrm{Ss}$. In addition, HR for HH seq was greater than for $H$ in only $12 / 20$ sessions (3/5 for J.P. and G.K., $2 / 5$ for D.H., and $4 / 5$ for J.A.). At the 2.0 - $\mathrm{sec}$ duration, there is very little difference between the accuracy of $\mathrm{H}$ and $\mathrm{HH}$ seq as measured by either HR or IT-in fact, IT values for HH seq were slightly less than for $H$ for each $S$.

The finding that the sequential repetition of brightness increased absolute judgment accuracy, while the sequential repetition of hue did not, is somewhat puzzling. However, verbal reports from Ss strongly indicate that a sensory artifact, color adaptation, may have contributed to this discrepancy. Ss complained that the appearance of the stimulus categories changed during the course of a set of 60 trials; in particular, the appearance of the green stimuli became increasingly yellow, or even brown, following the presentation of stimuli at the blue end of the continuum. ${ }^{4}$ This change in appearance was reportedly most severe in the HH seq condition with the 2.0-sec duration, and therefore may have counteracted any potential facilitation from multiple observations over time.

\section{Spatial Dimensional Redundancy}

The effect of the spatial presentation of the two correlated stimulus dimensions can be evaluted by comparing $\mathrm{HB}$ sim with the most discriminable single-dimension condition for each $S$. If it is assumed that $B$ was the most discriminable single dimension since HR was greater for $B$ than for $H$ for each $S$ at the $0.1-\mathrm{sec}$ duration, there is little evidence for a gain in accuracy with the spatial presentation of both dimensions. While slightly greater accuracy, as measured by either HR or IT, is noted for $3 / 4 \mathrm{Ss}$, it should be mentioned that the HR for HB sim was greater than it was for B in only 9/20 experimental sessions. These results support those of Lockhead (1966b), that little or no improvement in accuracy results from the simultaneous presentation of spatially separated redundant stimulus dimensions, at least for such brief exposure durations.

At the 2.0-sec duration, the effects of spatial dimensional redundancy are quite inconsistent across Ss, making interpretation of results difficult. For example, J.P.'s HR for HB sim was $9.3 \%$ better than for B, and his IT value for $\mathrm{HB}$ sim was .41 bits better than for B. ${ }^{5}$ Gains in accuracy of this size are close to those obtained in studies in which hue and brightness have been compounded into the same element (e.g., Eriksen \& Hake, 1955) and approach the theoretical maximum obtainable gain predicted by a normative model of information integration described by Garner and Lee (1962). On the other hand, much less improvement in accuracy was observed for the remaining three Ss, and J.A. demonstrated essentially no improvement in accuracy at all. It is possible that individual Ss, who are essentially untrained in psychophysical tasks of this type, may differ in their ability to apply a successful strategy for combining perceptual information from spatially separated stimulus dimensions, even when the exposure duration is as long as $2.0 \mathrm{sec}$. Thus, while the mean gain in IT for $\mathrm{HB}$ sim at the $2.0-\mathrm{sec}$ duration, approximately .2 bits in IT over $\mathrm{H}$ and $\mathrm{B}$, might suggest a substantial gain in accuracy, the fact that one $S$ contributed overwhelmingly to this result warrants considerable caution in interpretation.

\section{Sequential Dimensional Redundancy}

The effects of sequential dimensional redundancy can be evaluated by comparing $\mathrm{HB}$ seq with best single 
dimension for each S. At the 0.1-sec duration, all four Ss showed greater accuracy for $\mathrm{HB}$ seq than for either $\mathrm{H}$ or $B$, as measured by either IT or HR. If it is assumed that $B$ was the most discriminable single-dimension condition, the mean amount of improvement in IT, .15 bits, corresponds almost exactly with the value obtained by Lockhead (1966b) for an equivalent condition. Since, however, HB seq produced no greater accuracy than BB seq at the 0.1-sec duration, there is no evidence that sequential dimensional redundancy provided more facilitation of absolute judgment than the sequential repetition of identical stimuli.

At the 2.0-sec duration, HB seq produced the greatest accuracy of any condition for all four Ss as measured by either HR or IT. HR for HB seq was greater than for $\mathrm{H}$ in all five experimental sessions for each $S$, HB seq produced a greater HR than B in $4 / 5$ sessions for J.P. and D.H., and in all five sessions for the other two Ss. Mean HR for $\mathrm{HB}$ seq was $12.1 \%$ greater than for $\mathrm{H}$ and $10.8 \%$ greater than for $\mathrm{B}$; IT for HB seq is .37 bits better than for $\mathrm{H}$ and .39 bits better than for B. Thus, these gains in accuracy are considerably greater than those observed at the $0.1-\mathrm{sec}$ duration. Of particular interest is the extent to which $\mathrm{HB}$ seq produced greater accuracy than BB seq. Only $1 / 4$ Ss had a greater mean HR for HB seq than for $B B$ seq at the 0.1 -sec duration, while all four Ss had greater HRs for HB seq than for BB seq at the 2.0-sec duration. While the HR for HB seq was greater than for the BB seq in only $9 / 20$ sessions at the $0.1-\mathrm{sec}$ duration, HB seq produced a greater HR in $18 / 20$ sessions ( $3 / 5$ sessions for G.K., all five sessions for the other Ss) at the 2.0 -sec duration. ${ }^{6}$ It thus appears that the advantage of sequentially presented redundant dimensions over simple stimulus repetition is highly dependent upon either the exposure duration of the stimulus elements themselves or perhaps the total amount of time the $S$ has to separately encode each stimulus dimension.

\section{DISCUSSION}

\section{Stimulus Repetition}

The spatial repetition of identical stimuli did not facilitate absolute judgment of the stimulus dimensions used in this study. Previous research (e.g., Eriksen \& Lappin, 1965; Garner \& Flowers, 1969; Flowers \& Garner, 1971) has demonstrated that both speed of discrimination and accuracy of stimulus identification may be improved by spatial repetition of stimuli, but only when performance is limited by low stimulus visibility due to brief exposure and low contrast. Such performance limitations have been described by Garner (1970) as state limitations. In the present study, stimuli were presented at high contrast. Even at the $0.1-\mathrm{sec}$ duration, it is doubtful that the Ss would have made identification errors if only two stimulus categories had been used. According to Garner's (1970) terminology, performance was primarily process limited. The finding that no increase in absolute judgment accuracy resulted from spatial repetition of stimuli is in agreement with the results of previous studies in which spatial repetition of highly visible stimuli did not increase classification speed (e.g., Flowers \& Garner, 1971).

On the other hand, some previous studies have indicated that the sequential repetition of stimuli may produce a small improvement in the identification accuracy for highly visible (i.e., process limited) stimuli. For example, Halpern and Ulehla (1970) showed that the tilt discrimination of highly visible lines was improved by sequential stimulus repetition. These findings are consistent with the small increase in the absolute judgment accuracy of brightness, observed in the present study. The finding that sequential, but not spatial, repetition of stimuli is facilitating in such process-limited discrimination tasks may be the result of the partial independence over time, but not space, of certain organismic factors such as the state of attention and foveal fixation.

\section{Redundant Separable Dimensions}

The results of the present study demonstrate that correlated stimulus dimensions need not be integral and compounded in order to produce a substantial increase in accuracy of absolute judgment. HB seq at the $2.0-\mathrm{sec}$ duration produced IT values which range between .21 and .45 bits greater than those produced by the single dimension have the greatest IT value for each S. ${ }^{7}$ According to an information integration model described by Garner and Lee (1962), maximum possible gain in IT, assuming an ideally efficient integration of perceptual information from two perceptually independent stimulus dimensions should be about .48 bits, given that each dimension transmits about 2.5 bits when presented alone. This .48-bit prediction assumes, however, that an unlimited number of stimulus and response categories are available, and does not take into account the restriction on the room for improvement brought about by having only 15 categories. Flowers (1973) has suggested, through the use of a computer-simulated modification of the Garner and Lee model which allows for the 15-category constraint, that about .40 bits is a more reasonable estimate of the "ideal" gain in performance. If an integration or "averaging" process is, in fact, an appropriate description of how Ss use the redundant dimensions in Condition HB seq at the 2.0-sec duration, a rather efficient integration would therefore seem to be indicated by the magnitude of the observed gain in accuracy.

The finding that much less improvement in accuracy resulted from sequential dimensional redundancy at the 0.1 -sec exposure duration indicates that the provision of sufficient processing time is crucial for the efficient integration of information from the two dimensions. 
While it cannot be determined from the present data whether it is the presentation time for each dimension or the length of the interval between sequential stimulus presentations which is the most critical, the total time required for efficient integration would appear to be several seconds. One should not expect, therefore, sequential or spatial dimensional redundancy of the type used in the present study to facilitate performance in tasks in which stimuli are presented over brief durations. The necessity of this processing time probably arises from several factors, including the need for separate eye fixations, and perhaps the need to establish an efficient memory code for the value of each dimension.

\section{Information Integration vs Pattern Encoding}

The importance of processing time in Condition HB seq strongly suggests that some type of relatively time-consuming serial processing of the redundant dimensions is taking place, perhaps involving a combination or averaging of separately encoded response tendencies. The simple numeric averaging of response values is one possible mode of integration suggested by Garner and Morton (1969), although there is no direct evidence from the present study that Ss were using this type of overt arithmetic strategy. Other authors, such as Doherty and Keeley (1969), have suggested that Ss may behave as Bayesian predictors in combining successive stimulus judgments. While one may propose numerous other process models for the integration of separate stimulus judgments, it is almost impossible, as Garner and Flowers (1969) have pointed out, to determine the exact form of stimulus encoding on the basis of the simple fitting of data to a model.

A considerably different mode of processing separately presented correlated stimulus dimensions has been suggested in a study by Lockhead (1970). In this study, several absolute judgment experiments were conducted in which the values of two or more dimensions were completely correlated, but in a nonlinear manner such that the values of one stimulus dimension varied as a nonmonotonic "sawtooth" function of the other dimensions. This sawtooth redundancy produced much larger gains in absolute judgment accuracy than have been observed in previous studies which employed linear correlations between stimulus values; furthermore, these gains in accuracy far exceeded the predictions of models which assume an averaging or integration of perceptual information from each dimension (e.g., Garner \& Lee, 1962). When the dimensions of loudness and brightness were simultaneously presented for only $0.1 \mathrm{sec}$, sawtooth redundancy produced very large increases in accuracy. The linear pairing of these obviously separable dimensions, presented simultaneously at this duration, produced no improvement in performance, suggesting that the usefulness of the sawtooth redundancy is much less dependent upon stimulus presentation time than linear redundancy. Lockhead has argued that the sawtooth pairing of stimulus dimensions leads to the encoding of unique stimulus patterns based upon the relationship between values of the stimulus dimensions. Since sawtooth redundancy was very effective in increasing absolute judgment accuracy even when stimulus dimensions were simultaneously presented for rather brief durations, it appears that pattern encoding is a much more rapid mode of processing redundant dimensions than that of combining separately obtained judgments of linearly correlated stimulus values.

Thus, both pattern encoding and the combining of separately obtained stimulus judgments seem to be important modes of processing of correlated multidimensional stimulus information. The relative usefulness of these modes in perceptual discrimination and identification tasks would appear to depend upon both the form of the redundancy among the dimensions and the method of stimulus presentation.

\section{REFERENCES}

Doherty, M. E., \& Keeley, S. M. A Bayesian prediction of four-look recognition performance from one-look data. Perception \& Psychophy sics, 1969, 5, 362-364.

Eriksen, C. W., \& Hake, H. W. Multidimensional stimulus differences and accuracy of discrimination. Journal of Experimental Psychology, 1955, 50, 153-160.

Eriksen, C. W., \& Lappin, J. S. Internal perceptual system noise and redundancy in simultaneous inputs in form identification. Psychonomic Science, 1965, 2, 351-352.

Flowers, J. H. The effect of simultaneous and sequential presentation of stimulus dimensions on absolute judgment accuracy. (Doctoral dissertation, Yale University) Ann Arbor, Michigan: University Microfilms, 1973. No. 73-14,225.

Flowers, J. H., \& Garner, W. R. The effect of stimulus element redundancy on speed of discrimination as a function of state and process limitation. Perception \& Psychophysics, 1971, 9, 158-160.

Garner, W. R. The stimulus in information processing. American Psychologist, 1970, 25, 350-358.

Garner, W. R. Information integration and form of encoding. In A. W. Melton and E. Martin (Eds.), Coding processes in human memory. Washington, D.C: Winston, 1972.

Garner, W. R., \& Felfoldy, G. L. Integrality of stimulus dimensions in various types of information processing. Cognitive Psychology, 1970, 1, 225-241.

Garner, W. R., \& Flowers, J. H. The effect of redundant stimulus elements on visual discrimination as a function of element heterogeneity, equal discriminability, and position uncertainty. Perception \& Psychophysics, 1969, 6, 216-220.

Garner, W. R., \& Lee, W. An analysis of redundancy in perceptual discrimination. Perceptual \& Motor Skills, 1962, $15,367-388$.

Garner, W. R., \& Morton, J. Perceptual independence: Definitions, models, and experimental paradigms. Psychological Bulletin, 1969, 72, 233-259.

Halpern, J., \& Ulehla, Z. J. The effect of multiple responses and certainty estimates on the integration of visual information. Perception \& Psychophysics, 1970, 7, 129-132.

Lockhead, G. R. Effects of dimensional redundancy on visual discrimination. Joumal of Experimental Psychology, 1966a, $72,95-104$. 
Lockhead, G. R. Visual discrimination and methods of presenting redundant stimuli. Proceedings of the 74th Annual Convention of the American Psychological Association, $1966 \mathrm{~b}, 67-68$.

Lockhead, G. R. Identification and the form of discrimination space. Joumal of Experimental Psychology, 1970, 85, 1-10.

\section{NOTES}

1. IT values were computed from confusion matrices from all five sessions pooled ( 300 trials) for each combination of stimulus condition and exposure duration, since individual sessions provided ton few observations for meaningful calculations of the IT statistic.

2. A small Conditions by Duration interaction was noted for IT values, $F(7,21)=2.52, \mathrm{p}<.05$, but this interaction did not reach the .05 level for $H R$.

3. The mean HR and the mean IT value (across durations) were each significantly greater than for B, according to analysis of variance contrasts: $F(1,21)=8.21$ for $H R ; F(1,21)=11.33$ for IT, $\mathrm{p}<.01$.

4. This phenomenon of color change was also personally observed by the author following the experiment.

5. In addition, for J.P., HR for HB sim at the 2.0-sec duration was greater than for $B$ in all five experimental sessions.

6. The statistical significance of this interaction is supported by an analysis of variance contrast comparing the difference between the IT values for $\mathrm{HB}$ seq and $\mathrm{BB}$ seq at the 0.1-sec duration, with the difference between $\mathrm{HB}$ seq and $\mathrm{BB}$ seq at the $2.0-\sec$ duration, $F(1,21)=18.4, p<.01$.

7. This estimate of the gain in performance is actually conservatively biased, since the selection of the single dimension having the greatest IT value capitalizes on chance, particularly since $\mathrm{H}$ and $\mathrm{B}$ produced approximately equal performance at the 2.0-sec duration.

(Received for publication August 6, 1973; accepted September 24, 1973.) 